UCRL-JC-128008

PREPRINT

\title{
Absorptance Measurements of Transmissive Optical Components by the Surface Thermal Lensing Technique
}

\author{
R. Chow, J. R. Taylor, Z. L. Wu, \\ Yue Han, TianLi Yang
}

This paper was prepared for submittal to the Annual Symposium on Optical Materials for High Power Lasers Boulder, $\mathrm{CO}$

October 6-8, 1997

September 24, 1997

This is a preprint of a paper intended for publication in a journal or proceedings. Since changes may be made before publication, this preprint is made available with the understanding that it will not be cited or reproduced without the permission of the author. 


\section{DISCLAIMER}

This document was prepared as an account of work sponsored by an agency of the United States Government. Neither the United States Government nor the University of California nor any of their employees, makes any warranty, express or implied, or assumes any legal liability or responsibility for the accuracy, completeness, or usefulness of any information, apparatus, product, or process disclosed, or represents that its use would not infringe privately owned rights. Reference herein to any specific commercial product, process, or service by trade name, trademark, manufacturer, or other wise, does not necessarily constitute or imply its endorsement, recommendation, or favoring by the United States Government or the University of California. The views and opinions of authors expressed herein do not necessarily state or reflect those of the United States Government or the University of California, and shall not be used for advertising or product endorsement purposes. 
Absorptance measurements of transmissive optical components by the surface thermal lensing technique

\author{
Robert Chow ${ }^{a}$, John R. Taylor ${ }^{a}$, Zhou Ling Wu ${ }^{b}$, Yue Han ${ }^{b}$, and Tian Li Yang ${ }^{b}$ \\ aLawrence Livermore National Laboratory, Livermore, CA 94551 \\ bEastern Michigan University, Ypsilanti, MI 48197
}

\begin{abstract}
The surface thermal lensing technique (STL) successfully resolved and measured the absorptance of transmissive optical components: near-normal angle-of-incidence anti-reflectors and beam splitters. The STL system uses an Ar ion laser to pump the components at $514.5 \mathrm{~nm}$. The absorptance-induced surface deformation diffracts the $\mathrm{HeNe}$ probe beam into a photo-detector. The signal intensity was calibrated with a sample of known absorptance. The optical components were designed to function in a copper vapor laser (CVL) transport system, and were previously tested for absorptance with a high power CVL system at $511 \mathrm{~nm}$. To assure proper absorptance data from the STL system, the pump laser power densities were set at the operational level of the coatings, absorptance time trends were monitored, and absorptance area scans were made. Both types of transmissive optics are more stable than the CVL high reflectors that were measured in another study. Parameter studies based on Fresnel diffraction theory were also performed to optimize experimental condition. The STL system was assessed to have $10 \mathrm{ppb}$ sensitivity for absorption measurement given $2 \mathrm{~W}$ of pump power.
\end{abstract}

Key words: Absorptance, anti-reflector, beam splitter, laser optics, surface thermal lensing, photothermal

\title{
1. INTRODUCTION
}

A uranium enrichment plant utilizing atomic vapor laser isotope separation (AVLIS) technology is currently being planned by the United States Energy Corporation. Deployment of the AVLIS plant will require tens of thousands of high performance optical coatings. These coatings are expected to satisfy simultaneous performance of a number of material properties, one of which is extremely low levels of absorptance. Low absorptance coatings minimize thermally-induced distortion of the optic during exposure to a high irradiance. Otherwise, distortions affect the wavefront of the process laser beam and subsequently degrade the AVLIS plant process efficiencies. Low absorptance coatings also reduce the incidence of cw laser-induced damage. The current cycle for optimizing the deposition process for absorptance is done over a period of weeks, where the coating supplier makes test runs, sends the coated witnesses to LLNL for absorptance testing, and waits for the LLNL report before adjusting the coating design or the deposition process. The long time delays between test runs are not conducive to a manufacturing environment. A preferable method is to have an absorptance test instrument readily accessible to the coating supplier. This requires an instrument which is easy to operate, repeatable, reliable, and consists of commercially available lasers and optical components.

The photothermal deflection (PTD) technique appears suited for this absorptance application [1-3]. The technique has high sensitivity, uses low to moderate power lasers, and the coating may be tested at its design wavelength by selecting the appropriate pump laser. However, the technique involves the difficult steps of aligning two small diameter $(\sim 10$ to $100 \mu \mathrm{m})$ laser beams to close proximity in order to make the measurement. Coating-induced scatter makes the small beams appear larger so that critical positioning of the beams cannot be done visually. Also, the signal to noise ratio is low, requiring lownoise signal amplification between the detector and the lock-in amplifier. The surface thermal lensing (STL) configuration of the photothermal deformation technique is an alternate detection method $[4,5]$ that has demonstrated more precision and easier alignment than the conventional set-up [6]. The reflected signal in an STL configuration has been modeled, and demonstrated on high absorptance filter glass, low absorptance single layer refractive metal oxide layers [6], and low absorptance high reflectors [7]. The purpose of this work is to demonstrate that STL can resolve the absorptance of low absorptance transmissive optics.

\section{EXPERIMENT}

The absorptance measurement by the PTD technique is illustrated in Figure 1. The absorption of the pump beam energy causes a corresponding local temperature rise of the sample, which leads to a local surface deformation due to thermal expansion. By probing the laser-induced surface deformation, whose magnitude along the perpendicular direction to the 
sample surface can be less than $0.1 \mathrm{~nm}$, one can relate the detected signal to the optical and thermo-mechanical properties of the sample. Conventional PTD uses a second, tightly focused, laser beam (probe beam) on the deformed surface as the sensing method. In the PTD set-up, the probe beam size is much smaller than that of the lateral dimension of the deformed area. Thus after reflection from the deformed surface, the probe beam experiences a change in direction which is proportional to the slope of the surface deformation. For the STL set-up, in contrast to PTD, a probe beam with a diameter equal to or larger than the lateral dimension of the thermally deformed area is reflected from the sample surface. The deformed area on the sample surface acts as a lens which diffracts the probe beam. The shape of the surface deformation is thus recorded in the diffraction pattern of the reflected probe beam, which can be analyzed by using either a CCD camera or a scanning photodetector.

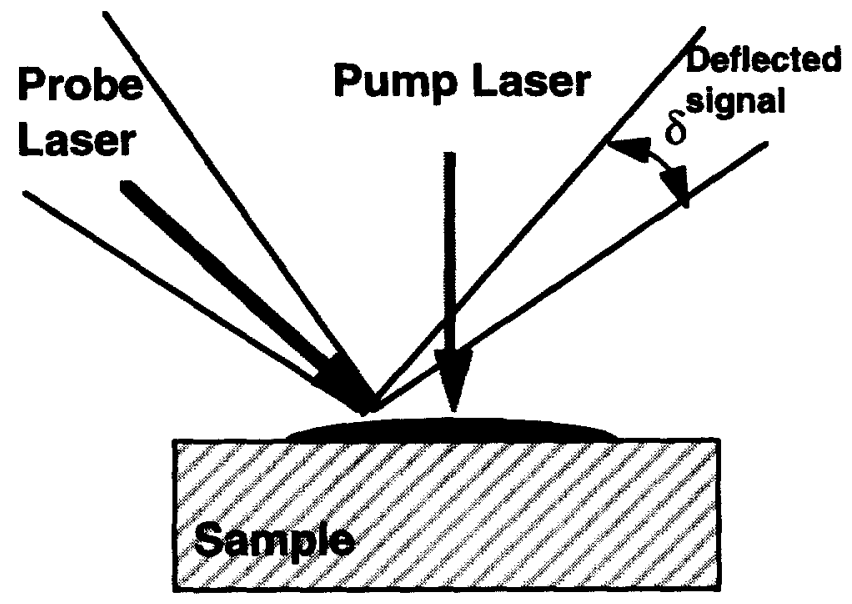

Photothermal Deformation

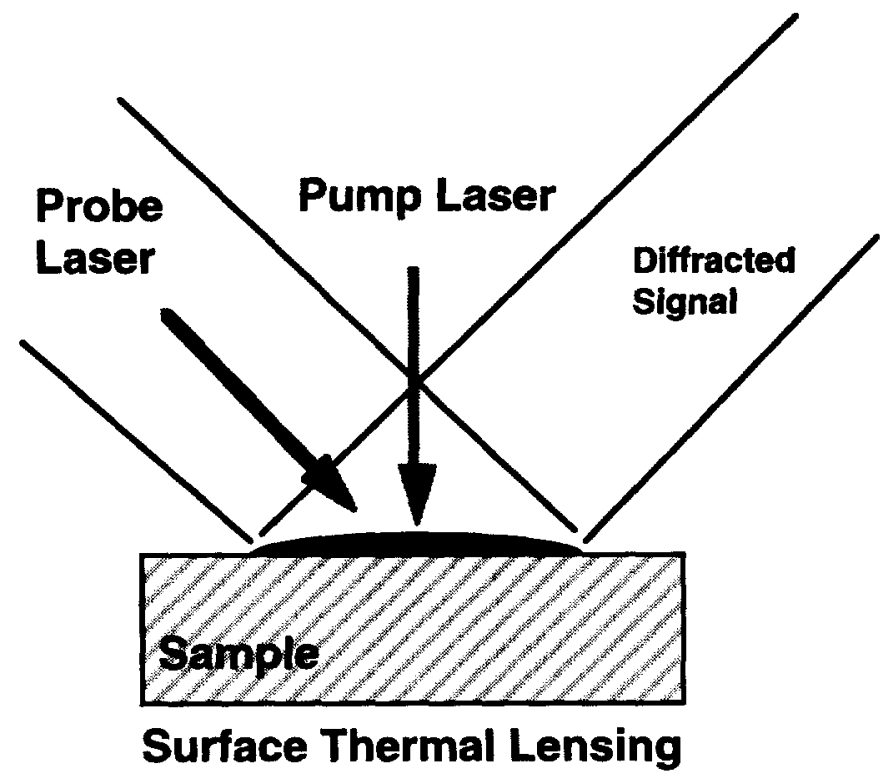

Figure 1 Comparison of a conventional photothermal deflection (PTD) and the surface thermal lensing (STL) technique. The PTD requires two small diameter laser beams aligned close to each other. The probe beam diameter must be smaller than the lateral dimension of the thermally-induced deformation. The STL technique uses a probe beam diameter that is greater than the lateral dimension of the deformation.

STL offers two advantages over the conventional PTD configuration. STL has the same high sensitivity but avoids the critical alignment requirements of PTD. STL is easier to align because the probe beam is larger than the pump beam. When probe beam overlaps the pump beam, a diffraction-induced signal is obtained, and the signal may then be optimized. In the present work, both the pump and probe beam are in the visible wavelength region and observable to the unaided eye. Furthermore, if a CCD camera is used for detecting the diffraction pattern, STL obtains the full field information of the surface deformation. This can be an important advantage over PTD, where the probe beam samples only a small spot of the deformed area. Therefore for the STL technique, the time to record the shape of the deformation is significantly reduced since no scanning procedure is needed.

The STL experimental setup for the absorptance measurements is illustrated in Figure 2. An Ar ion laser at $514.5 \mathrm{~nm}$ is used as the pump source. The beam is split so that the power can be measured, modulated at $12 \mathrm{~Hz}$ with a mechanical chopper wheel synchronized to a lock-in amplifier, and focused onto the sample at near normal angle of incidence with a beam diameter about $100 \mu \mathrm{m}$. The probe laser beam is from a $10 \mathrm{~mW}$ HeNe laser, at the wavelength of $632.8 \mathrm{~nm}$. It is focused onto the sample surface, coincident with the pump laser beam. For this particular set-up, the He-Ne laser beam diameter is focused to a diameter of about $500 \mu \mathrm{m}$ to achieve optimum sensitivity of this set-up. The detector is located 60 $\mathrm{cm}$ from the sample. To detect the STL signal, a pinhole is placed in front of the photodetector. In this way the detector monitors intensity changes at the center of the probe beam, which is proportional to the optical absorptance of the sample. To convert the PTD signal into absorptance data, the traditional calibration method for PTD is used, where the calibration coefficient is determined from the PTD signal of a thin film sample with known absorptance $[2,3,8]$. 
The coatings for the absorptance measurements are transmissive coatings designed to reflect wavelengths of 511 and $578 \mathrm{~nm}$. Specifically, three anti-reflectors and seven beam splitters were tested in this study. The beam splitters have transmittances ranging from $14 \%$ to $50 \%$. All the coatings are made by a reactive e-beam process and deposited onto superpolished fused silica substrates. The substrates are $76.2 \mathrm{~mm}$ in diameter and about $15 \mathrm{~mm}$ thick. The coatings have various material combinations and layer thicknesses.

The absorptances were measured by the STL technique described above and by a laser radiometric technique using a custombuilt copper vapor laser (CVL) as the pump source [9]. The CVL is a high-average-power (600 watts), $4.4 \mathrm{kHz}$ repetition rate, $50 \mathrm{~ns}$ pulse-width system [10]. The CVL beam is filtered to $511 \mathrm{~nm}$ light and focused approximately into a $2 \mathrm{~mm}$ diameter spot at 10 degrees angle of incidence onto the coating. An Inframetrics (Model 525) infrared camera senses the temperature differential with respect to the rest of the surface. The temperature change is converted into an absorptance value using an empirically derived absorptance model [11]. This radiometric technique has been used successfully for the past decade to assist coating suppliers in the optimization of their respective coating processes and designs.

A measurement procedure was followed that replicates the operating conditions of the coating:

1. The samples were cleaned prior to exposure to the pump beam;

2. the $514.5 \mathrm{~nm}$ Ar ion laser light was picked off from a prism expander to minimize absorptance from extraneous wavelengths;

3. the power density was set to a value between 2 and $5 \mathrm{~kW} / \mathrm{cm}^{2}$; and

4. increase the area tested for absorptance.

A sample translation stage, a computer controller and data acquisition package was added to the STL set-up so that large area scanning could be done on a routine basis. In addition, the sample stage was located within an HEPA filtered enclosure to minimize particulate contamination during a scan. The purpose of scanning was to be able to sample more of the coating and not rely on a single (or even a few) $100 \mu \mathrm{m}$ diameter spot measurement to represent the coating absorptance. A $1 \mathrm{~mm}^{2}$ area was scanned since this is similar to the area sampled by the CVL-pumped method at LLNL. To account for time dependent absorptance behavior and avoid inordinately long scan times, the scanning strategy was to dwell on each site long enough to gather the initial time dependent behavior, and return to a typical site to perform the long term absorptance behavior measurement. Based on the absorptance work with high reflectors [7], the initial dwell time per site was set to about one second and the long term dwell time $\geq 3 \mathrm{~min}$.

\section{STL MODELING}

Fresnel diffraction theory was used to model the beam intensities in the conventional PTD and STL configurations [12, 13]. The results given in Fig. 3 are the three dimensional beam profiles incident upon the detector. In the conventional PTD configuration, a pump beam diameter of $25 \mu \mathrm{m}$ and a probe beam diameter of $5 \mu \mathrm{m}$ was used. A surface deformation deflects the beam from the center of the detector to one side. In the STL configuration, a pump beam of $25 \mu \mathrm{m}$ diameter and a probe beam of $50 \mu \mathrm{m}$ was used. The presence of a surface deformation of the same magnitude creates a doughnut shaped beam profile. With this modeling capability, the sensitivity of the STL signal can be studied with respect to different materials and the sensitivity to configurational variations. For instance in the last panel, the signal onto the detector is skewed because the probe beam is no longer on-axis with the pump beam. 


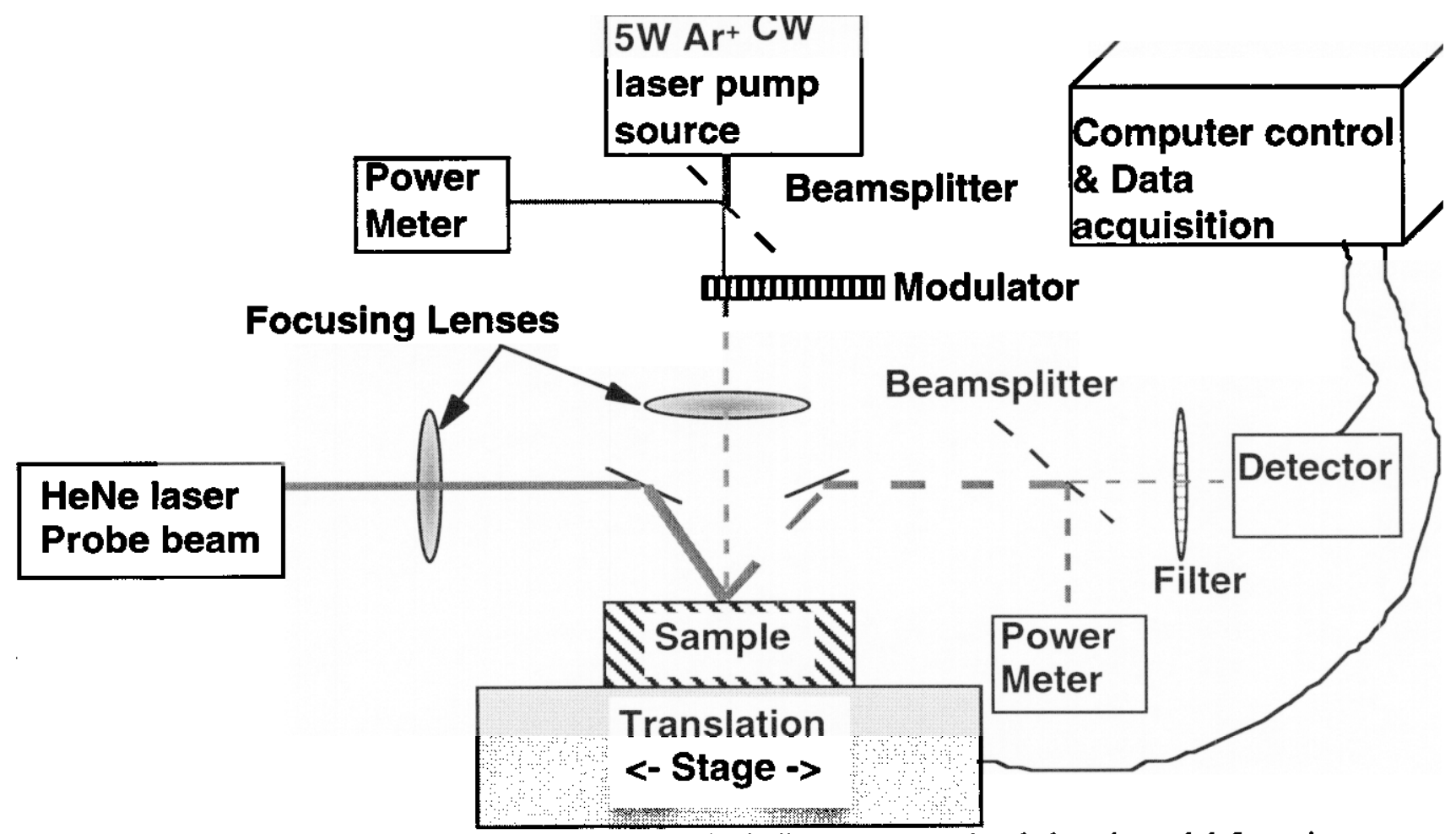

Figure 2. The surface thermal lensing set-up. The set-up is similar to a conventional photothermal deformation setup. The probe beam diameter at the surface is $500 \mu \mathrm{m}$, and the pump beam diameter is $100 \mu \mathrm{m}$.

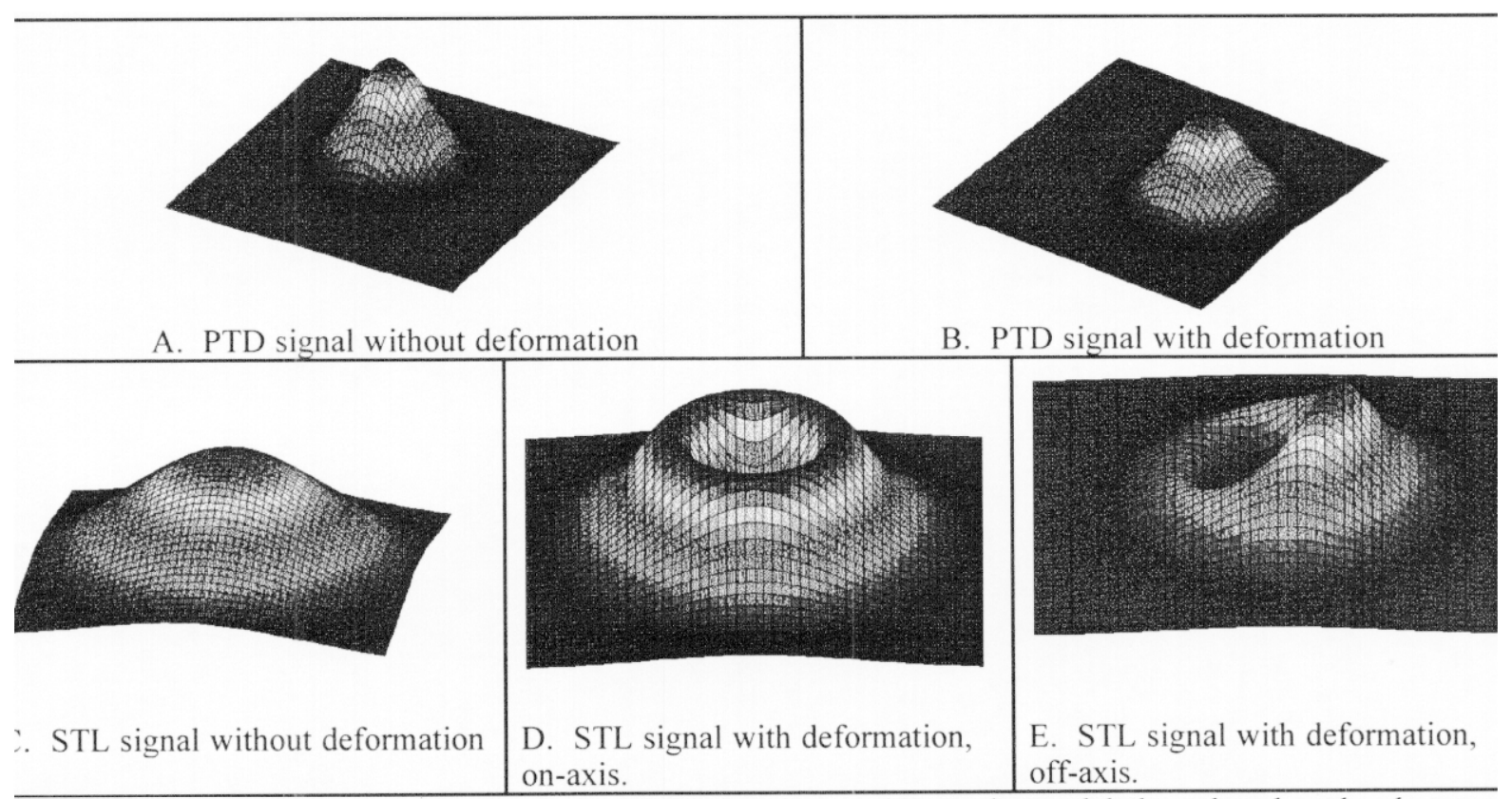

igure 3 Simulated beam intensities at the detector. Panels A and B are the modeled results when the absorptal e is measured with a conventional photothermal deformation set-up. Panel $A$ is the beam profile without a surface $d$ ormation. Panel B is the beam profile with a surface deformation. Note that the bump deflects the beam within the detector. Panels C, $\mathrm{D}$, and $\mathrm{E}$ are the results when a surface thermal lensing configuration is used. Panel $\mathrm{C}$ had a larger beam profile because the surface thermal lensing configuration uses a larger probe beam. Panel D is the effect of a surface bump of the same magnitude as in panel $\mathrm{B}$. Panel $\mathrm{E}$ is the beam profile when the probe beam is not on-axis with the surface deformation. 


\section{RESULTS}

\subsection{Coating absorptance homogeneity}

Five areas distributed along $2.4 \mathrm{~cm}$ of a high reflector sample radius were scanned. Each scanned area was $1 \mathrm{~mm}^{2}$. The statistics in Table 1 show that the scanned areas are indistinguishable in absorptance from each other. We conclude that absorptance measurements collected within a $1 \mathrm{~mm}^{2}$ area adequately represents the absorptance of the entire sample. We will also assume that the absorptance is uniform on a macroscopic scale for high performance optical coatings and therefore the
scan area may be selected randomly on the sample.

Table 1 Absorptance values from the same sample. Five different $1 \mathrm{~mm}^{2}$ areas were measured on one sample. The standard deviation is a combination of both the precision of the measurement and the non-uniformity of the coating absorptance.

\begin{tabular}{|c|c|c|c|c|c|}
\hline Site & 1 & 2 & 3 & 4 & 5 \\
\hline Mean Signal Level & 157.7 & 151.5 & 154.3 & 148.4 & 152.2 \\
\hline Standard Deviation & 43.4 & 35.0 & 35.1 & 38.9 & 34.8 \\
\hline
\end{tabular}

\subsection{Anti-reflector coatings}

The STL technique was able to resolve low absorptance CVL anti-reflective (AR) coatings. Three AR CVL coatings were tested for absorptance. The samples were designed to the same reflectance specifications but made in three different runs. Figure 4 is a chart of absorptance on the $Y$-axis and the sample tested along the X-axis. Scan \#1 and \#2 are STL measurements at two different locations of the same sample. The Spot measurement is when the computer returned to a typical site and made a long term ( 5 minute) absorptance scan. The absorptances were stable for all three of these ARs. The absorptances measured from the CVL-pumped set up are shown as the fourth bar of the sample set. In this case, the data between these two techniques correlates well even though the absolute values differ. Figure 5 shows the scanned images from these ARs. The sites where there are groups of saturated pixels (light) may be an indication of high absorbing defects. he coatings either have a low defect density or have defects below the spatial resolution of the STL set-up.

\section{Low Absorptance Anti-reflectors}

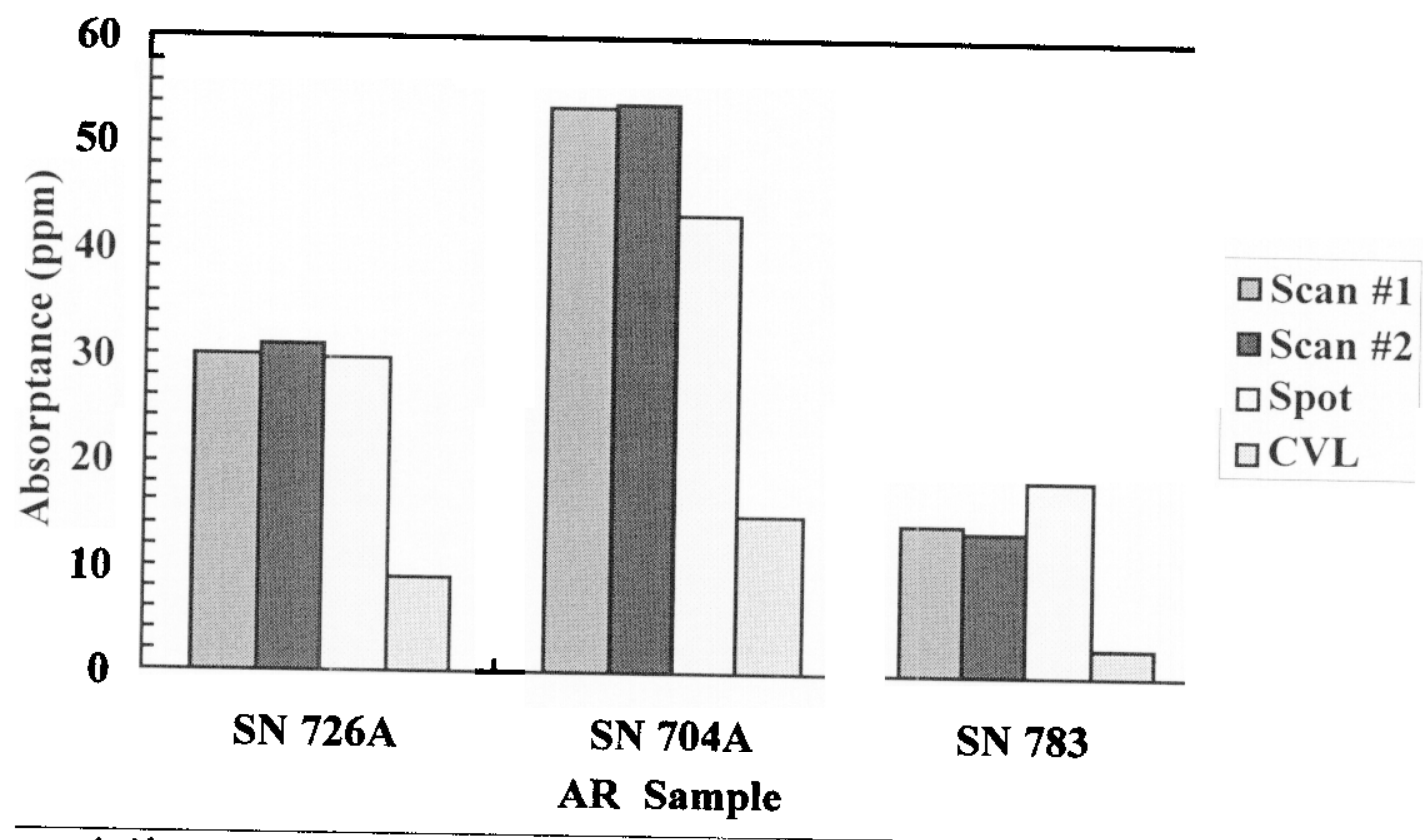

gure 4 Absorptances of anti-reflectors. Scans \#1 and \#2, and the Spot are measurements made by $t$ :STL technique. The ot measurement is the long term absorptance value at one site. The absorptances determined by e CVL technique are ven by the fourth column for each sample. 


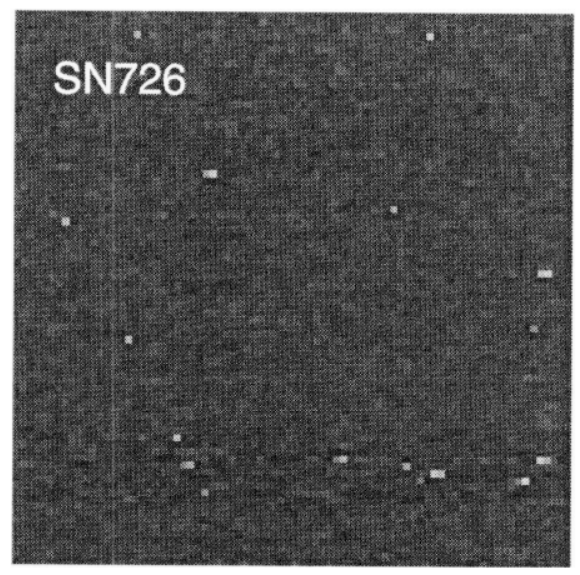

Figure 5 Anti-reflector absorptances by the STL technique. There are low densities of high absorbing defects. The scan dimensions are $1 \mathrm{~mm} \times 1 \mathrm{~mm}$.
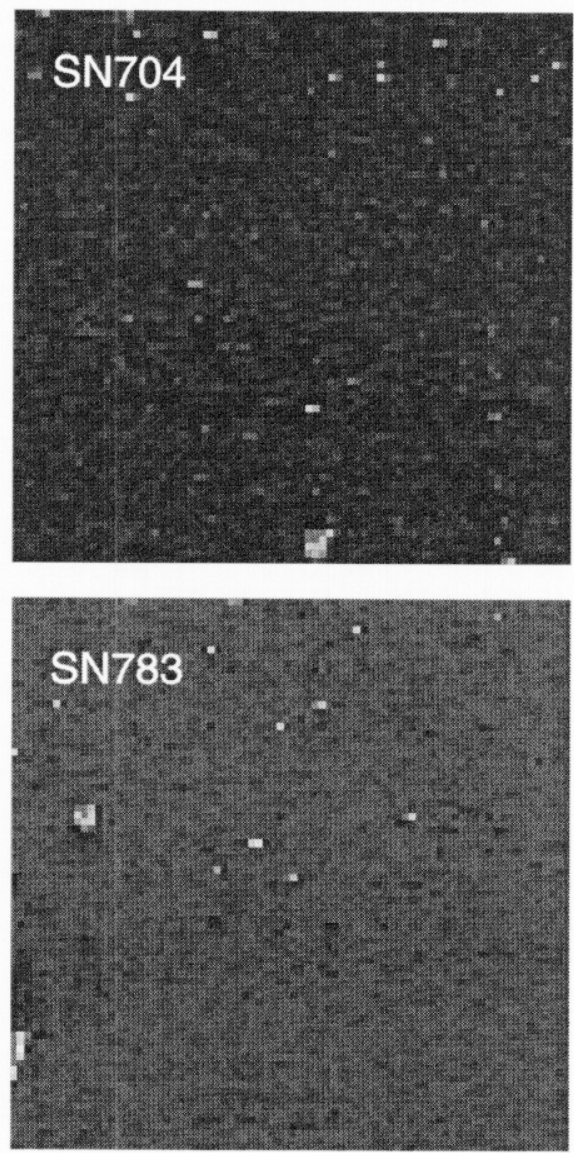

\subsection{Beam splitter coatings}

The STL technique was able to resolve low absorptance CVL beam splitter coatings. Seven beam splitters with various transmittance from $14 \%$ to $50 \%$ were measured. Figure 6 is a chart of absorptance on the Y-axis in Log scale and the sample tested on the X-axis. For each sample, the first column is the absorptance measured from an STL scan, the second column is the STL absorptance measurement taken from one spot irradiated for a long time (300 seconds), the third column is the absorptance measured by the CVL-pump technique, and in the fourth space is a notation regarding the defect size found within the STL scan area (SN 355 and 356 also include an absorptance value in the fourth column). An observation from this chart is that the long term STL absorptance value is either equal to (as in the case of SN 79) or less than that value determined by the CVL-pumped technique. Figure 7 are the scan images from the beam splitters. The scans show that most of these coatings have high absorbing defects with areas larger than those observed with the ARs and beam splitter SN 79. 
The other observation is that the samples with a discrepancy between the STL and CVL absorptance values are the same samples with large area high absorbing defects. The defect absorptance of samples SN 355 and 356 are given as the fourth bar to show how high the STL absorptances are for these sites. The arrow on SN 355 indicates the absorptance is greater
than $300 \mathrm{ppm}$.

\section{DISCUSSION}

The STL absorptance values may be lower than that of the CVL technique for the beam splitters for two reasons. The first reason is that the high absorbing defects may not be accounted properly in the data acquisition and analysis. When a high absorbing defect is encountered, a high signal level sent to the lock-in amplifier may saturate the amplifier because its gain is set too low. In that case, the lock-in sends to the computer the value of its upper limit range, not the real signal amplitude. A simple procedure is to set the signal recording level a lower gain to minimize lock-in amplifier saturation. Also, the long term absorptance behavior of a high absorbing defect site may be more significant than that from the low absorbing area. In addition to having a high absorptance, the defect could have an absorptance that increases to a stable value, or worst yet, increases with time until damage occurs. To determine the significance of this effect, the data acquisition software should be modified to return to a typical and a high absorbing site, and make the long term absorptance measurement at both locations. There may even be a size threshold upon which a defect area become the significant contributor to the overall coating absorptance. Comparing STL and CVL absorptances, the high absorbing defects in the AR coatings and in beam splitter SN 79 scans did not appear to contribute to the absorptance as much as those samples with larger area defects.

The second reason to consider is that these techniques measure different physical factors. The CVL technique measures the infrared emission of the irradiated area on the coating surface. The CVL technique assumes that the emissivity of the coating is constant with material and temperature. The STL technique measures the deformation of the substrate. This effect involves absorption by the coating, thermal conduction of the energy to the substrate, and the creation of the surface bump. The thermal transport to the substrate depends on the thermal properties of a multilayered coating, which involves a number of layers, and various layer thicknesses, materials, and interfaces. In both cases, the absorption is a crucial part of the mechanism that produces a detectable phenomenon but absorptance is not directly measured.

\section{Low Absorptance Beam Splitter}

10000

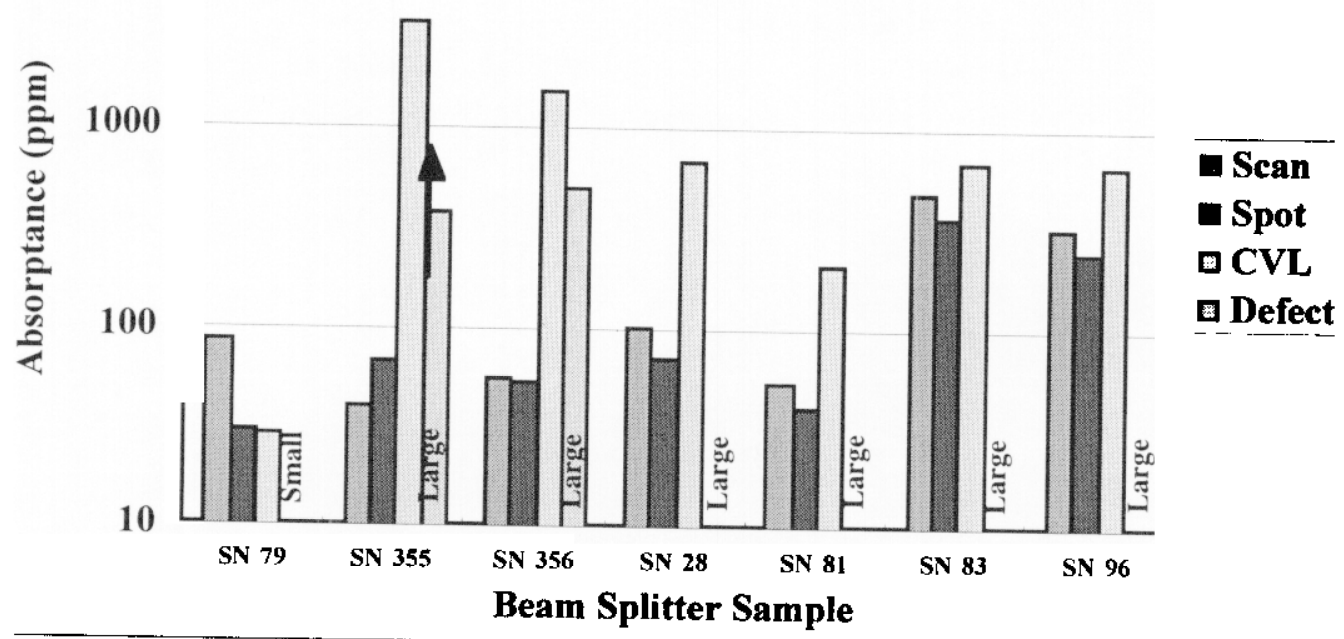

igure 6 Beam splitter absorptances. Scan and Spot absorptances are measured by the STL $t$ hnique and are the first and second bars, respectively. The third bar is the absorptance measured by the CVL pump tec] ique. The fourth bar is the absorptance of a high absorber site found during a STL scan and/or a notation to the size of arrow on SN 355 indicates that the absorptance is greater than $300 \mathrm{ppm}$. ie relative defect area. The 

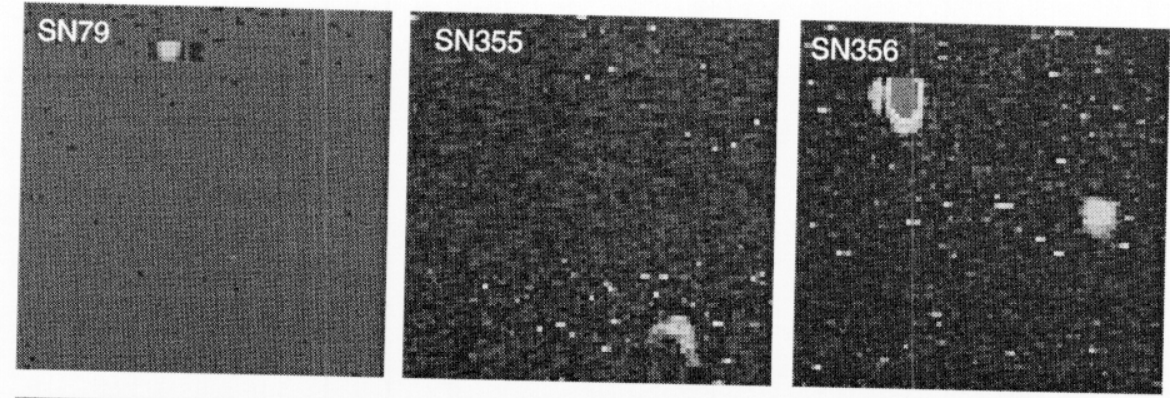

Figure 7 Absorptance images of beam splitters

The scan dimensions are $1 \times 1 \mathrm{~mm}$. The high absorbing defects have larger areas than those found in the AR coatings and beam splitter SN 79
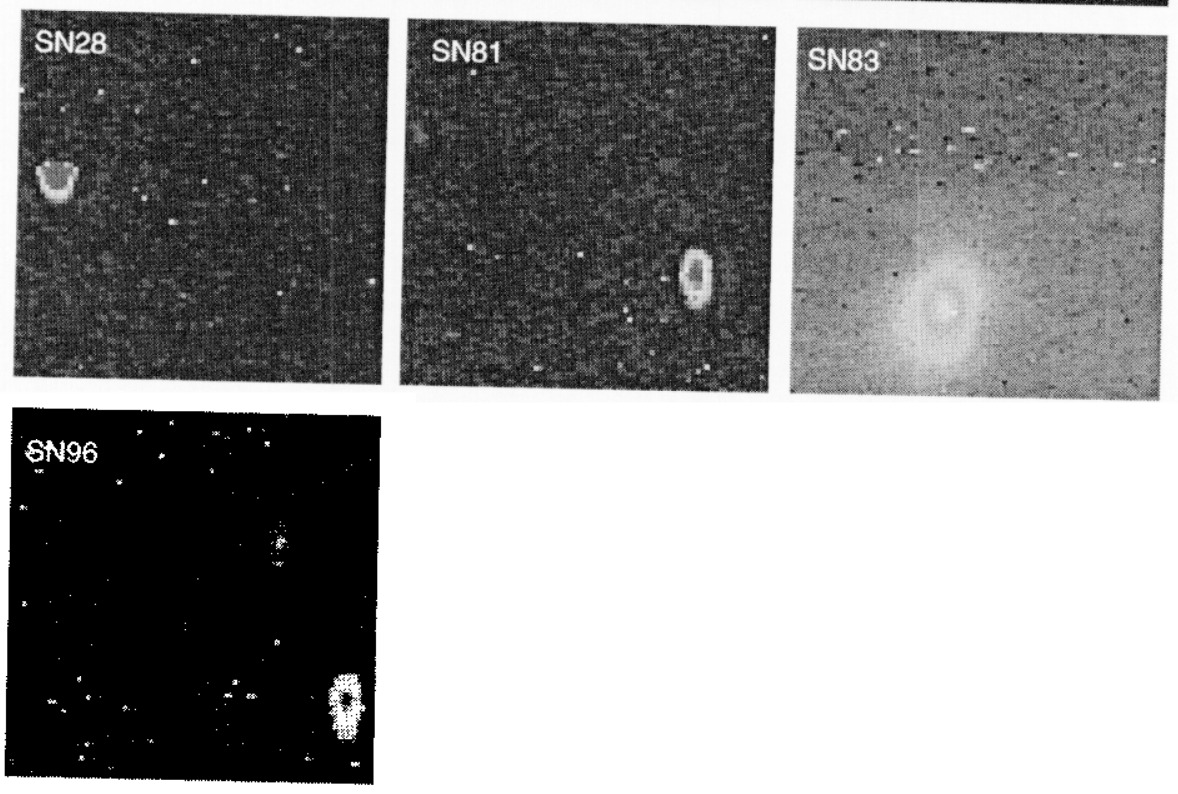

\section{SUMMARY}

The STL technique has adequate resolution to measure absorptances of transmissive, refractory metal oxides, multilayered The STL system has a computer controlle upgraded with scanning capabilities to accommodate a $76.2 \mathrm{~mm}$ diameter sample. is contained in an HEPA filtered enclosure to minimize stage, data acquisition, and data analysis software. The sample stage Fresnel diffraction theory was performed on the PTD technique in tse during absorptance measurements. Modeling based on (he PTD technique in the conventional and STL configuration.

The next generation STL system is in the design phase. We are planning to incorporate features that enable absorptance testing of other AVLIS optical coatings, and possibly a laser survivability capability. The software program will be revised
to handle high absorbing defect sites

\section{AUSPICES} This work was performed under the auspices of the U. S. Department of Energy by Lawrence Livermore National Laboratory
under contract No. W-7405-Eng-48.

\section{REFERENCES}

1. M. A. Olmstead, N. M. Amer, S. Kohn, D. Founire, and A. C. Boccara, "Photothermal displacement spectroscopy: an
optical probe for solids and surfaces," Appl. Phys. A 32, 1983, 141-154. 2. E. Welsh and D. Ristau, "Photothermal measurements on optical thin films," Applied Optics, v34, 1 Nov. 1995, 7239-
7253. 
3. Z. L. Wu, M. Thompsen, P. K. Kuo, Y. S. Lu, C. J. Stolz, M. R. Kozlowski, "Overview of photothermal

characterization of optical thin film coatings," in Laser-induced damage in optical materials: 1995, SPIE vol 2714, 465-481.

4. P. K. Kuo and M. Munidasa, Review of Progress in Quantitative NDE, edited by D. O. Thompsen and D. E. Chimenti, Plunum New York, 8A, 627-633, 1989; P. K. Kuo and M. Munidasa, "Single beam interferometry of a thermal bump," Appl. Opt. 29, 5326, 1990.

5. H. Saito, M. Irikura, M. Haraguchi, and M. Fukui, "New type of photothermal spectroscopic technique," Appl. Opt. 31, 2074, 1992.

6. Z. L. Wu, P. K. Kuo, Y. S. Lu, and S. T. Gu, "Laser induced thermal lensing for thin film characterization," in Laserinduced damage in optical materials: 1995, SPIE vol 2714, 294-303, 1996.

7. R. Chow, J. R. Taylor, Z. Wu, R. Krupka, T. Yang, "High reflector absorptance measurements by the surface thermal lensing technique," in Laser-induced damage in optical materials: 1996, SPIE vol 2966, 354-361, 1996.

8. Z. L. Wu, S. T. Gu, P. K. Kuo, Y. S. Lu, and Rene Krupka, "Non-destructive evaluation of thin film coatings using surface thermal lensing technique," to be published in Thin Solid Films.

9. C. R. Stolz, J. R. Taylor, T. Sarginson, "Damage test capabilities using a high-repetition rate visible laser at LLNL," in Laser-induced damage in optical materials: 1991, SPIE v1624, 109-115.

10. Richard Hackel and Bruce Warner, "The copper-pumped dye laser system at LLNL," SPIE OE/LASE'93, Los Angeles, CA, Jan. 19, 1993.

11. V. G. Draggoo, R. G. Morton, R. H. Sawicki and H. D. Bissinger, "Optical coating absorption measurement for high power laser systems," in High Power and Solid State Lasers, W. Simmons, ed., Proc. Soc. Photo-Opt. Instrum. Eng., vol $622,1986,186-190$.

12. Y. Han, Q. Zhao, and Z. L. Wu, "Near-field detection of laser-induced thermal waves in optical materials," in Laserinduced damage in optical materials: 1997, SPIE vol 3244, to be published in 1998.

13. J. Power, "Pulsed mode thermal lens effect detection in the near field via thermally-induced probe beam spatial phase modulation: theory," Appl. Optics, 29, 52-63(1996). 


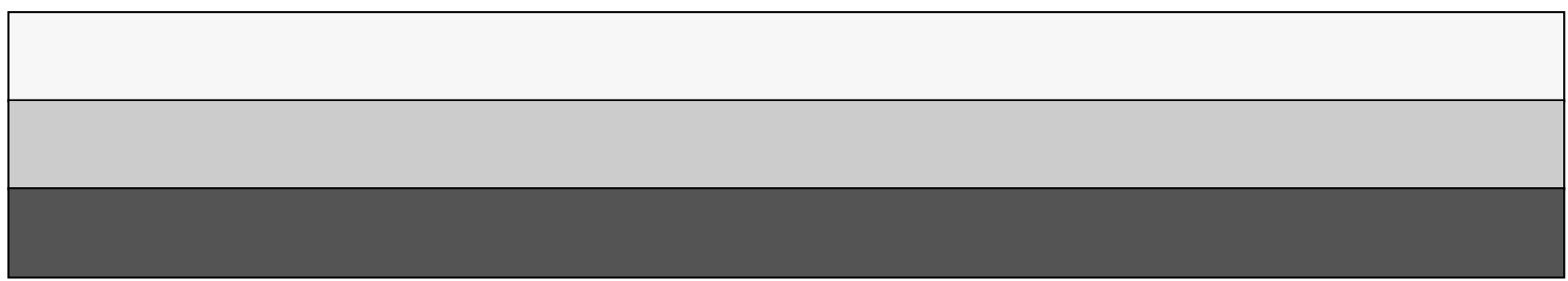

